\title{
PREVALÊNCIA DE OBESIDADE EM IDOSOS LONGEVOS E SUA ASSOCIACCÄO COM FATORES DE RISCO E MORBIDADES CARDIOVASCULARES
}

\author{
Ivana Beatrice Mânica Da Cruz*, Marilia Siqueira Campos Almeida, Carla Helena Augustin Schwanke, \\ EmílIo HideYukı Moriguch \\ Trabalho realizado no Instituto de Geriatria e Gerontologia da Pontifícia Universidade \\ Católica do Rio Grande do Sul (IGG-PUCRS), Porto Alegre, RS.
}

RESUMO - OBjetivos. Avaliar a prevalência de obesidade e sua associação com fatores de risco e morbidades cardiovasculares em idosos longevos (com idade $\geq 80$ anos) residentes em VeranópolisRS, Brasil.

Métodos. Participaram do estudo 196 idosos (69 homens e 127 mulheres), $91 \%$ da população $\geq 80$ anos até julho de 1996. Para avaliação e classificação da obesidade, utilizou-se 0 índice de massa corporal (IMC) e os critérios diagnósticos da Organização Mundial da Saúde (OMS) e do National Health and Nutrition Examination Survey (NHANES III). Os fatores de risco cardiovascular investigados foram sexo, idade, hipertensão arterial sistêmica (HAS), hipercolesterolemia, hipertrigliceridemia, diabetes mellitus (DM) e tabagismo. Nas morbidades cardiovasculares, considerou-se a história de infarto agudo do miocárdio (IAM), claudicação intermitente e acidente vascular encefálico (AVE). A relação cintura-quadril $(\mathrm{C} / \mathrm{Q})$, o consumo regular de bebidas alcoólicas e a atividade física regular também foram investigados.
Resultados. A prevalência de obesidade foi $23,3 \%$ pelo critério da OMS (sem diferença entre os sexos, $p=0,124$ ) e $45,6 \%$ pelo critério NHANES III (significativamente maior entre as mulheres, $p=0,05$ ). Mulheres obesas apresentaram maiores níveis de pressão arterial sistólica e glicose, menores níveis de HDL-c e maior freqüência de HAS e DM; já os homens obesos apresentaram maiores níveis de pressão arterial diastólica, colesterol total, LDL-c e maior freqüência de hipercolesterolemia. As médias de $C / Q$ e de triglicerídios, bem como a freqüência de hipertrigliceridemia foram maiores nos obesos de ambos os sexos.

Conclusöes. A prevalência de obesidade entre os idosos longevos foi alta e sua associação com os fatores de risco cardiovascular foi sexo-dependente. Em relação às morbidades, não se observou diferença entre os indivíduos obesos e não obesos.

UnITERMOs. Obesidade. Fatores de risco cardiovascular. Morbidade cardiovascular. Idosos longevos.

\section{INTRODUÇÃO}

Com o fenômeno de envelhecimento populacional observado neste último século', aumenta, cada vez mais, a necessidade de conhecimento dos fatores que incidem sobre a prevalência das doenças crônico-degenerativas associadas à idade. A partir desta perspectiva, em 1994, foi implementado um projeto de pesquisa no Instituto de Geriatria e Gerontologia da Pontificia Universidade Católica do Rio Grande do Sul (IGG-PUCRS) sobre envelhecimento bem sucedido denominado Projeto Veranópolis. Na primeira fase da investigação (1994-1997), foram avaliados idosos com idade $\geq 80$ anos. Veranópolis foi escolhida uma vez que tal município apresenta uma expectativa média de vida ao nascer similar às observadas em países desenvolvidos e industrializados ${ }^{2}$ como os Estados Unidos

* Correspondência

Av. Ipiranga, $6690-3^{\circ}$ andar

CEP: $90610-000$ - Porto Alegre - RS
(76,7 anos) e o Japão $(80,9 \text { anos })^{3}$. Na década de 90 a expectativa média de vida ao nascer em Veranópolis era de 77,7 anos ${ }^{4}$, enquanto que no Rio Grande do Sul e no Brasil, nesta mesma época, as expectativas médias giraram em torno de 72 anos e 67 anos, respectivamente $^{5}$. A Tabela I sintetiza os principais indicadores demográficos e socioeconômicos e de saúde do municípió.

Análises preliminares dos idosos longevos de Veranópolis apontaram a obesidade como um dos fatores de risco cardiovascular mais prevalentes ${ }^{7}$. Taxas elevadas de sobrepeso e obesidade em todas as faixas etárias, atingindo os dois sexos, estão sendo observadas no mundo inteiro. Uma das causas deste fenômeno está relacionada ao aumento significativo na ingestão de gordura e açúcar e com a diminuição do consumo de fibras e carboidratos complexos nestas últimas décadas ${ }^{8}$. A OMS, no seu relatório de 2002, apontou a obesidade como uma epidemia, encontrando-se no topo de uma lista dos 10 riscos para a saúde humana?.
No Brasil, o aumento da prevalência da obesidade começou a ser observado paralelamente à mudança econômica e epidemiológica ocorrida entre as décadas de 1960 e 1990. Dois estudos transversais sobre a condição nutricional brasileira foram feitos e têm servido como referência para a avaliação dos níveis de obesidade no País: o Estudo Nacional da Despesa Familiar (ENDEF), realizado em 1974-75 ${ }^{10}$ e a Pesquisa Nacional sobre Saúde e Nutrição (PNSN)" realizada em 1989. Uma nova análise dos dados obtidos nestes dois estudos, realizada por Monteiro ${ }^{10}$ mostrou que adultos entre 25 e 64 anos de idade tiveram um aumento na prevalência de obesidade entre 1974 e 1989 de 5,5\% para 9,6\%.

Apesar deste contexto, o papel da obesidade como risco cardiovascular em idosos longevos até recentemente não era tão claro quanto em indivíduos adultos e idosos jovens $^{12,13,14}$.

Por tal motivo, em países desenvolvidos, estudos longitudinais começaram a ser 


\begin{tabular}{|c|c|}
\hline \multicolumn{2}{|c|}{ Tabela I - Características gerais do Município de Veranópolis-RS 6} \\
\hline Características & \\
\hline $\begin{array}{l}\text { Ano de criação } \\
\text { Economia } \\
\text { PIB (US\$) } \\
\text { Regiäo oeográíca } \\
\text { Área(Km) } \\
\text { População } \\
\text { Expectativa de vida ao nascer (anos) } \\
\quad \text { Masculina } \\
\text { Feminina } \\
\text { Coeficiente de mortalidade infantil ( I00 mil hab) } \\
\text { Coeficiente de mortalidade geral (I00 mil hab) } \\
\text { Número de idosos longevos (> } 80 \text { anos) } \\
\text { Mortalidade total ( } 1996) \\
\text { Principais causas da mortalidade [n (\%)] } \\
\text { Aparelho circulatório } \\
\text { Neoplasias } \\
\text { Aparelho respiratório } \\
\text { Causas externas } \\
\text { Outras causas } \\
\end{array}$ & $\begin{array}{c}1884 \\
\text { Indústria } \\
\text { | } 48.987 .147 \\
\text { Serra } \\
306,3 \\
18.122 \\
77,7 \\
72,1 \\
83,8 \\
0,001 \\
5,59 \\
213 \\
107 \\
\\
31(29) \\
25(23) \\
8(7) \\
16(15) \\
27(26) \\
\end{array}$ \\
\hline
\end{tabular}

Fontes: Núcleo de Informação de Saúde-RS (1997), Instituto Brasileiro de Geografia e Estatísica. Dados referentes aos anos de 1996 el 1997.

conduzidos na tentativa de estabelecer se a obesidade continuaria sendo um fator preditor forte de doenças cardiovasculares em idosos longevos. Este é o caso da investigação recentemente conduzida por Nybo et al. ${ }^{15}$ em 2.249 nonagenários da Dinamarca. O estudo investigou fatores preditores de mortalidade nesta população, e os resultados sugerem que a obesidade, assim como o tabagismo e fatores socioeconômicos, perdem importância na predição de mortes neste grupo etário. Entretanto, se for considerado que os idosos longevos investigados vivem em um país desenvolvido, cujas condições socioeconômicas e do sistema de saúde são satisfatórias, é difícil fazer uma extrapolação dos resultados para países em desenvolvimento como o Brasil.

Portanto, investigações que analisem a obesidade em idosos longevos brasileiros são necessárias. Dentro deste contexto, o objetivo geral deste estudo foi avaliar a prevalência de obesidade e sua associação com fatores de risco e morbidades cardiovasculares em idosos longevos (com idade $\geq 80$ anos) residentes em Veranópolis-RS.

\section{Métodos}

O presente estudo foi do tipo transversal, observacional, analítico e de base populacional envolvendo indivíduos idosos longevos
( $\geq 80$ anos), realizado a partir de dados obtidos em 1996 por uma equipe multidisciplinar. A identificação dos participantes foi feita a partir da recontagem populacional realizada pelo Instituto Brasileiro de Geografia e Estatística (IBGE) em 1996, obtida na Secretaria do IBGE do município. Foram identificados 213 moradores incluídos neste grupo etário sendo que 193 concordaram em participar do estudo (91\%). Por ser uma população que permaneceu estável ao longo dos últimos trinta anos, com um número total de habitantes oscilando entre 17-20 mil pessoas no período, e por terem os voluntários uma idade avançada, a taxa de perda por outro motivo, que não o óbito, foi considerada nula entre 1994 e 1996. A coleta de dados incluiu avaliação clínica, antropométrica e bioquímica associadas ao risco cardiovascular (fatores de risco clássicos e emergentes) e presença de morbidades cardiovasculares prévias. Detalhes da coleta dos dados clínicos e laboratoriais são descritos em Schwanke et al. ${ }^{16}$, incluindo anamnese, eletrocardiograma (ECG), análises bioquímicas e medida de pressão arterial. Além dos exames físicos, foi realizada uma entrevista estruturada aplicada aos voluntários na presença do seu cuidador (respondente próximo). Caso o idoso não se recordasse e/ ou tivesse dificuldade para relatar as questões solicitadas, a pergunta era novamente direcionada ao cuidador.

Diagnóstico da obesidade: os idosos foram classificados como obesos e não obesos através do índice de massa corporal (IMC), produto da divisão do peso corporal pela altura ao quadrado $\left(\mathrm{P} / \mathrm{A}^{2}\right)$. Dois critérios diagnósticos foram utilizados: ${ }^{\circ}$ - critério estabelecido pela Organização Mundial de Saúde (OMS) para avaliação de população adulta e idosa ${ }^{17}$ e também utilizado nos estudos do PNSN ${ }^{18}$ - magreza grave $(\mathrm{IMC}<16,0)$, magreza moderada $(16,0-16,9)$, magreza leve $(17,0-18,4)$, adequado $(18,5-24,9)$, sobrepeso $(25,0-29,9)$, obesidade I $(30,0-39,9)$ e obesidade II (IMC $\geq$ 40); $2^{\circ}$ - critério estabelecido pelo National Health and Nutrition Examination Survey (NHANES III), que preconiza valores de IMC $\geq 27,8$ para classificar indivíduos masculinos como obesos e IMC $\geq 27,3$ para classificar indivíduos femininos como obesos ${ }^{19}$.

Variáveis investigadas: detalhes sobre a coleta das variáveis investigadas são descritos em Schwanke et al..$^{14}$ As principais variáveis investigadas foram: I) variáveis antropométricas e fisiológicas: peso, altura, a relação cintura/quadril (C/Q) e a pressão arterial; 2) bioquímicas: níveis de colesterol total, HDLcolesterol, triglicerídeos e glicose, medidos em $\mathrm{mg} / \mathrm{dL}$, foram obtidos através de técnicas enzimáticas colorimétricas e LDL-colesterol, através da fórmula de Friedewald; 3) variáveis do estilo de vida: prática regular de atividade física (o grau de atividade física foi inferido a partir do cálculo do gasto energético em quilocalorias por semana (Kcal/sem) relatado na entrevista), o consumo regular de bebida alcóolica e o tabagismo; 4) morbidades cardiovasculares: história prévia de infarto agudo do miocárdio (IAM), história prévia de acidente vascular encefálico (AVE) e relato de claudicação intermitente $(\mathrm{Cl})$.

Os dados foram estatisticamente analisados através do programa estatístico SPSS, versão 9.0. A seguir, as variáveis contínuas (idade, pressão arterial, perfil lipídico e glicemia) foram analisadas quanto à normalidade pelo teste de Kolmogorov-Smmirnoff. No caso do estudo, todas as variáveis apresentaram distribuição normal, sendo então comparadas entre obesos e não obesos homens e mulheres pelo teste paramétrico $t$ de Student. Os demais dados categóricos foram comparados por teste 
não-paramétrico do qui quadrado ou pelo teste exato de Fisher. Ao longo do estudo, o teste do qui quadrado foi expresso como $\chi^{2}$, enquanto os graus de liberdade como gl. Foram consideradas diferenças significativas valores de $p \leq 0,05$. Cálculo da razão de chance (OR) e do intervalo de confiança a 95\% (IC95\%) foram feitos. Análise multivariada adicional foi também realizada utilizando-se regressão logística, método Foward Wald. As variáveis incluídas na equação foram aquelas com $p<0,20$.

O estudo realizado foi aprovado pela Comissão Científica do Hospital São Lucas da PUCRS e pelo Comitê de Ética em Pesquisa da PUCRS, tendo seguido toda as recomendações da resolução 196/96 do Conselho Nacional de Saúde do Ministério da Saúde.

\section{Resultados}

A prevalência de obesidade, pelos critérios de classificação da OMS, foi de 23,3\%. Considerando estes critérios, a prevalência de sobrepeso e obesidade do tipo I (IMC entre 25 e $39,9 \mathrm{Kg} / \mathrm{m}^{2}$ ) foi alta (59\%), sendo igual a $55 \%$ nos homens e a $62 \%$ nas mulheres. Não foram observados indivíduos com magreza grave ou obesidade do tipo II em ambos os sexos, como pode ser observado na Figura I. A distribuição da obesidade não mostrou diferenças significativas entre os sexos considerando os critérios de classificação da OMS (Figura I) $\left(\chi^{2}=5,74 ; g=3\right.$; $p=0,124$ ).

Utilizando o critério do NHANES III, a prevalência de obesidade foi de 45,6\%, sendo que ocorreu uma prevalência significativamente maior de obesidade nas mulheres (5l\%) do que nos homens (36\%) $\left(\chi^{2}=3,833, g=1 ; p=0,05\right)$.

A análise da associação entre obesidade, fatores de risco e morbidades cardiovasculares foi feita utilizando-se o critério de diagnóstico da obesidade proposto pela OMS (Tabela 2).

Tanto a relação C/Q quanto os níveis de triglicerídios foram mais elevados em indivíduos obesos de ambos os sexos. Em mulheres obesas, a pressão arterial sistólica e os níveis plasmáticos de glicose foram mais elevados enquanto que os de HDL-c foram menores. Entre os homens obesos, a pressão arterial diastólica e os níveis plasmáticos de colesterol

Figura I -A) percentis observados do índice de massa corporal (IMC) de idosos homens e mulheres longevos ( 80 anos ou mais) residentes no município de Veranópolis-RS; $B$ ) distribuição das categorias do IMC entre os idosos homens e mulheres com 80 anos ou mais do município de Veranópolis-RS. Mag.= magreza
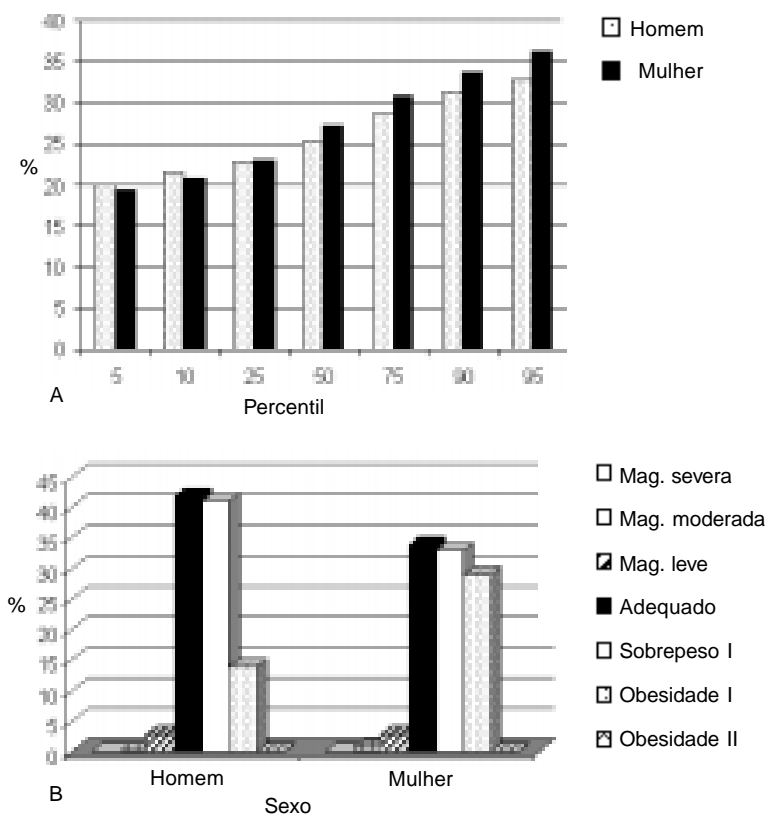

\begin{tabular}{|c|c|c|c|c|c|}
\hline \multirow[t]{2}{*}{ Variáveis } & \multirow[t]{2}{*}{ Grupos } & \multicolumn{2}{|c|}{ Homens } & \multicolumn{2}{|c|}{ Mulheres } \\
\hline & & média $\pm d p$ & $p$ & média $\pm d p$ & $p$ \\
\hline Idade(anos) & $\begin{array}{l}\mathrm{NO} \\
\mathrm{Ob}\end{array}$ & $\begin{array}{l}83,98 \pm 3,70 \\
83,11 \pm 3.11\end{array}$ & $p=0,157$ & $\begin{array}{l}83,91 \pm 3,26 \\
83,40 \pm 3,06\end{array}$ & $p=0,526$ \\
\hline PASistólica & NO & $154,92 \pm 19,04$ & $p=0,103$ & $170,22 \pm 24,10$ & $p=0,00$ \\
\hline $\begin{array}{l}(\mathrm{mmHg}) \\
\text { PADiastólica }\end{array}$ & $\mathrm{Ob}$ & $\begin{array}{r}166,67 \pm 26,53 \\
8699+1065\end{array}$ & $n=000$ & $\begin{array}{l}177,22 \pm 25,79 \\
888+1477\end{array}$ & $n=0150$ \\
\hline $\begin{array}{l}\text { PADiastólica } \\
\text { (mmHg) }\end{array}$ & $\begin{array}{l}\mathrm{NO} \\
\mathrm{Ob}\end{array}$ & $\begin{array}{l}86,99 \pm 10,65 \\
91,65 \pm 12,30\end{array}$ & $p=0,00$ & $\begin{array}{l}88,81 \pm 14,77 \\
95,68 \pm 13,08\end{array}$ & $p=0,150$ \\
\hline & $\begin{array}{l}\mathrm{NO} \\
\mathrm{Ob}\end{array}$ & $\begin{array}{l}0,93 \pm 0,05 \\
0,99 \pm 0,04\end{array}$ & $p=0,00$ & $\begin{array}{l}0,85 \pm 0,06 \\
0,88 \pm 0,05\end{array}$ & $p=0,01$ \\
\hline $\begin{array}{l}\text { Colesterol Total } \\
(\mathrm{mg} / \mathrm{dL})\end{array}$ & $\begin{array}{l}\mathrm{NO} \\
\mathrm{Ob}\end{array}$ & $\begin{array}{l}190,39 \pm 45,32 \\
217,53 \pm 40,81\end{array}$ & $p=0,01$ & $\begin{array}{l}219,81 \pm 53,38 \\
214,86 \pm 46,59\end{array}$ & $p=0,16$ \\
\hline HDL-c(mg/dl) & $\begin{array}{l}\mathrm{NO} \\
\mathrm{Ob}\end{array}$ & $\begin{array}{l}43,44 \pm 12,25 \\
42,56 \pm 14,02\end{array}$ & $p=0,145$ & $\begin{array}{l}50,23 \pm 11,47 \\
44,47 \pm 13,39\end{array}$ & $p=0,01$ \\
\hline LDL-c(mg/dL) & $\begin{array}{l}\mathrm{NO} \\
\mathrm{Ob}\end{array}$ & $\begin{array}{l}\mid 22,78 \pm 39,07 \\
|60,6| \pm 36,55\end{array}$ & $p=0,00$ & $\begin{array}{l}145,37 \pm 49,32 \\
\mid 38,53 \pm 40,67\end{array}$ & $p=0,231$ \\
\hline Triglicerídios & NO & $\begin{array}{r}120,88 \pm 39,07 \\
15068+8277\end{array}$ & $p=0,04$ & $121,03 \pm 37,47$ & $p=0,00$ \\
\hline Glicose(mg/dL) & $\begin{array}{l}\text { No } \\
\text { Ob }\end{array}$ & $\begin{array}{l}90,39 \pm 20,02 \\
97,96 \pm 27,55\end{array}$ & $p=0,|3|$ & $\begin{array}{l}92,52 \pm 19,58 \\
103,27 \pm 35,04\end{array}$ & $p=0,03$ \\
\hline
\end{tabular}

$\mathrm{NO}=$ indivíduos não obesos; $\mathrm{Ob}=$ indivíduos obesos; $\mathrm{dp}=$ desvio padrão; $p=$ significância do teste $\mathrm{t}$ de Student; $\mathrm{C} / \mathrm{Q}=$ relação cintura/quadril.

total e LDL-c foram mais elevados. O segundo grupo de variáveis estudadas foram as relacionadas com o estilo de vida dos idosos. Os resultados obtidos são apresentados na Figura 2.
A comparação na prevalência do hábito de fumar entre idosos obesos e não obesos foi significativa entre os homens $\left(\chi^{2}=7,018\right.$, $g \mid=1, p=0,04)$. No caso, nenhum homem 
Figura 2 - Distribuição das variáveis relacionadas ao estilo de vida entre homens e mulheres com 80 anos ou mais do município de Veranópolis-RS: (A) freqüência de indivíduos tabagistas; (B) frequeência de indivíduos que consomem bebidas alcóolicas regularmente; (C) frequiência de indivíduos que relatam prática regular de atividade física. $p=$ =nivel de significância do teste qui quadrado; $N S=$ não significativo
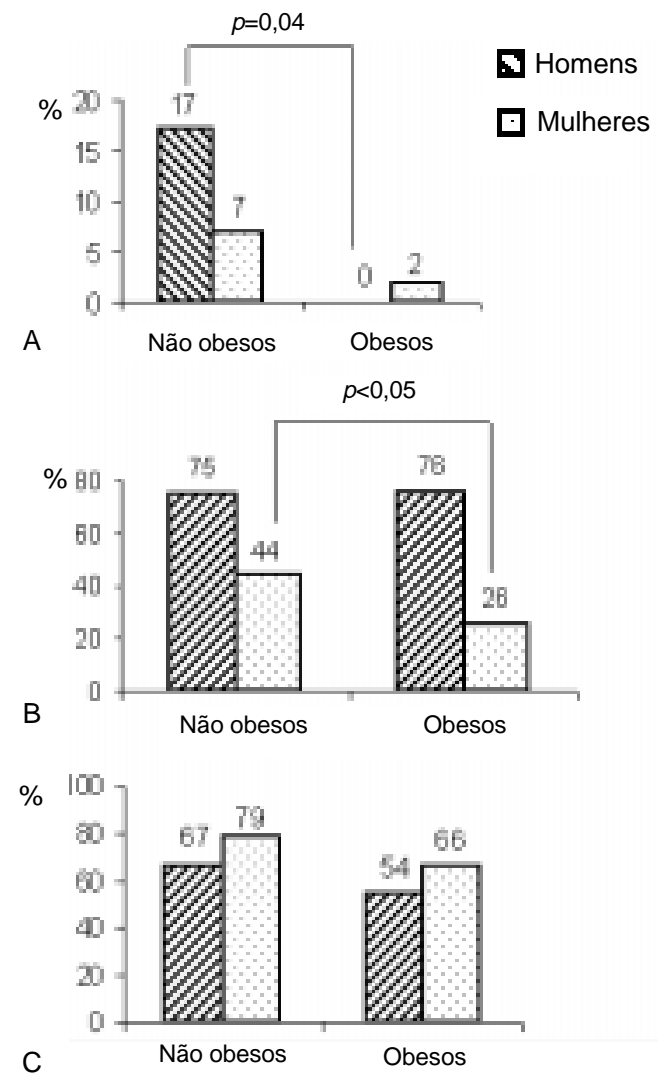

Tabela 3 - Comparação dos fatores de risco e morbidades cardiovasculares em idosos com 80 anos ou mais classificados quanto à obesidade, residentes em Veranópolis-RS

\begin{tabular}{|c|c|c|c|c|c|c|c|}
\hline \multirow[t]{2}{*}{ FR/morbidades } & \multirow[t]{2}{*}{ Grupos } & \multicolumn{3}{|c|}{ Homens } & \multicolumn{3}{|c|}{ Mulheres } \\
\hline & & $n$ & $\%$ & $p$ & $n$ & $\%$ & $p$ \\
\hline HAS & NO & $\begin{array}{l}38 \\
27\end{array}$ & $\begin{array}{l}86 \\
88\end{array}$ & 0,845 & 55 & $\begin{array}{l}90 \\
98\end{array}$ & 0,01 \\
\hline Hipercolesterolemia & $\begin{array}{l}\mathrm{NO} \\
\mathrm{Ob}\end{array}$ & $\begin{array}{l}4 \\
8\end{array}$ & $\begin{array}{l}10 \\
28\end{array}$ & 0,043 & $\begin{array}{l}17 \\
19\end{array}$ & $\begin{array}{l}28 \\
30\end{array}$ & 0,779 \\
\hline Hipertrigliceridemia & $\begin{array}{l}\mathrm{NO} \\
\mathrm{Ob}\end{array}$ & $\begin{array}{l}1 \\
6\end{array}$ & $\begin{array}{l}2 \\
24\end{array}$ & 0,04 & $\begin{array}{l}2 \\
14\end{array}$ & $\begin{array}{c}3 \\
23\end{array}$ & 0,000 \\
\hline Diabetes mellitus & $\begin{array}{l}\mathrm{NO} \\
\mathrm{Ob}\end{array}$ & $\begin{array}{l}4 \\
2\end{array}$ & $\begin{array}{l}10 \\
8\end{array}$ & 0,832 & $\begin{array}{l}5 \\
15\end{array}$ & $\begin{array}{c}9 \\
25\end{array}$ & 0,02 \\
\hline $\begin{array}{l}\text { Claudicação } \\
\text { intermitente }\end{array}$ & $\begin{array}{l}\mathrm{NO} \\
\mathrm{Ob}\end{array}$ & $\begin{array}{l}4 \\
2\end{array}$ & $\begin{array}{l}9 \\
8\end{array}$ & 0,876 & $\begin{array}{l}5 \\
4\end{array}$ & $\begin{array}{l}8 \\
6\end{array}$ & 0,682 \\
\hline AVE & $\begin{array}{l}\mathrm{NO} \\
\mathrm{Ob}\end{array}$ & $\begin{array}{l}2 \\
2\end{array}$ & $\begin{array}{l}5 \\
9\end{array}$ & 0,562 & $\begin{array}{l}2 \\
3\end{array}$ & $\begin{array}{l}3 \\
3\end{array}$ & 0,674 \\
\hline IAM & $\begin{array}{l}\mathrm{NO} \\
\mathrm{Ob}\end{array}$ & | & $\begin{array}{l}2 \\
4\end{array}$ & 0,617 & $\begin{array}{l}3 \\
2\end{array}$ & $\begin{array}{l}5 \\
3\end{array}$ & 0,621 \\
\hline
\end{tabular}

$\mathrm{NO}=$ indivíduos não obesos; $\mathrm{Ob}=$ indivíduos obesos; $\mathrm{n}=$ tamanho da amostra; $p=$ significância do teste não-paramétrico do qui-quadrado; $\mathrm{FR}=$ fatores de risco; $\mathrm{HAS}=$ hipertensão arterial sistêmica; $\mathrm{AVE}=$ acidente vascular encefálico; $\mid \mathrm{AM}=$ infarto agudo do miocárdio. obeso fumava. Mulheres não apresentaram diferenças significativas na prevalência do tabagismo entre obesas e não obesas $\left(\chi^{2}=2,347\right.$, $g|=|, p=0, \mid 25)$.

Considerando o relato de consumo regular de bebidas alcoólicas, mulheres obesas consumiram com menor freqüência bebidas alcoólicas do que as não obesas. Entretanto, este padrão não foi observado no sexo masculino. Dada a grande variação na quantidade média de álcool ingerida por semana, a mesma não diferiu entre os grupos analisados. No caso, em média, homens não obesos consumiram 271,99 $\pm 222,90 \mathrm{~g} / \mathrm{sem}$, homens obesos consumiram 280,9| $\pm|7|, 4 \mid \mathrm{g} /$ sem, mulheres não obesas consumiram $123,45 \pm 116,15 \mathrm{~g} / \mathrm{sem}$ e mulheres obesas consumiram I 16,54 $\pm 88,90 \mathrm{~g} / \mathrm{sem}$.

$A$ atividade física não apresentou diferenças significativas entre obesos e não obesos de ambos os sexos ( $\chi^{2}$ para homens $=1,006 ; g \mid=1 ; p=0,916 ; \chi^{2}$ para mulheres $=2,602 ; g l=1 ; p=0,145)$. A atividade física média semanal realizada pelos idosos, que indica o nível de gasto energético, também não mostrou diferenças significativas entre obesos e não obesos. No caso, homens não obesos apresentaram uma atividade física média de $5817,83 \pm 5431,74 \mathrm{kcal} / \mathrm{sem}$, enquanto a média dos homens obesos foi de 5883 , $18 \pm 5585,38 \mathrm{kcal} / \mathrm{sem}$. Já as mulheres não obesas tiveram uma atividade física média de 5903,91 $\pm 4342,50 \mathrm{kcal} / \mathrm{sem}$, enquanto, entre as obesas, a média foi de 4547 , $33 \pm 3930,78 \mathrm{kcal} / \mathrm{sem}$. Foram investigadas as associações sexo-dependentes entre obesidade, fatores de risco e morbidades cardiovasculares (Tabela 3).

Nos homens, ocorreu associação entre hipercolesterolemia e obesidade. Nas muIheres, idosas obesas apresentavam maior freqüência de hipertensão arterial e diabetes mellitus. Já a hipertrigliceridemia foi maior em indivíduos obesos de ambos os sexos. Não houve associação estatisticamente significativa entre as morbidades investigadas (IAM, AVE e $\mathrm{Cl}$ ) e a obesidade tanto em homens quanto em mulheres.

A análise multivariada mostrou que as associações observadas foram independentes. 


\section{Discussão}

Como previamente descrito por Michelon e Moriguchi ${ }^{7}$, a prevalência de obesidade foi alta na população investigada, ocorrendo associação sexo-espećíica com fatores de risco e morbidades cardiovasculares.

Tavares e Anjos ${ }^{16}$, a partir da análise do perfil antropométrico da população idosa brasileira realizada no estudo PNSN, onde foram investigados um total de 4.277 idosos (7,2\% da amostra total participante) apontaram para uma prevalência de obesidade de 5,7\% nos homens e de $18,2 \%$ nas mulheres considerando todos os idosos $\geq 60$ anos, ocorrendo aumento na prevalência da obesidade nos idosos que vivem na região sul do Brasil. Entretanto, no estudo de Tavares e Anjos ${ }^{18}$, apenas 1,2\% dos homens e $8,3 \%$ das mulheres idosos com $\geq 80$ anos foram considerados obesos. $O$ estudo recente feito por Abrantes et al. ${ }^{14}$ nas populações da região nordeste e sudeste do Brasil encontrou uma prevalência de sobrepeso de $16,5 \%$ e 27,2\% para homens e mulheres com idade igual ou superior a 80 anos, respectivamente. Quanto à obesidade, os autores observaram uma prevalência de 0,9\% nos homens e 5,7\% nas mulheres. As prevalências descritas por estes autores foram muito menores do que as descritas no presente estudo.

Considerando estes resultados, pode-se sugerir que os idosos de Veranópolis apresentaram um perfil de prevalência da obesidade mais similar ao de países desenvolvidos, como os Estados Unidos e da Europa, do que do Brasil como um todo ${ }^{14}$.

Resultados americanos obtidos pelo NHANES III apontaram um padrão de maior prevalência de obesidade em mulheres do que em homens idosos com 70 anos ou mais ${ }^{20}$. No caso, 2.917 indivíduos investigados, a prevalência de obesidade entre os homens foi $17,4 \%$ e entre mulheres foi $21,1 \%$ das idosas americanas. Investigações em homens longevos americanos ( $\geq 80$ anos) apresentaram 18\% de obesidade e mulheres, 26,2\% ${ }^{21}$. Ou seja, os idosos de Veranópolis-RS seriam discretamente menos obesos e as idosas um pouco mais obesas em comparação com idosos americanos da mesma faixa etária.

A associação entre obesidade, fatores de risco para aterosclerose e morbidades cardiovasculares também foi investigada por Alencar et al. ${ }^{12}$ em um estudo transversal em 516 idosos com média de idade de 75,6 anos atendidos em ambulatório na cidade de São Paulo. Os autores não encontraram associação entre obesidade e complicações de aterosclerose (doença arterial coronariana, acidente vascular encefálico e claudicação intermitente). Já Gruen e Connor ${ }^{13}$ no The Rancho Bernardo Studyavaliaram idosos com média de idade de 80 anos e seus resultados sugeriram que a relação C/Q não seria um método adequado para avaliar a obesidade, tanto em homens quanto em mulheres nesta faixa etária. Marafon et al. ${ }^{6}$ em um estudo que seguiu os idosos longevos de Veranópolis por um período de três anos (1996-1999), não encontraram associação entre IMC, relação C/Q e obesidade com mortalidade. Os autores comentaram que os resultados obtidos, que foram similares aos descritos em outros estudos internacionais envolvendo longevos, parecem apontar para a perda do poder da obesidade como fator de risco para doenças cardiovasculares. Ao contrário, eles discutem que existiriam evidências mostrando que a perda de peso estaria relacionada com uma maior carga de morbi-mortalidade nesta faixa etária.

A relação $\mathrm{C} / \mathrm{Q}$, outro parâmetro utilizado para detectar obesidade, no caso a central, também esteve aumentada entre os indivíduos obesos de Veranópolis-RS em ambos os sexos. Contudo, somente entre as mulheres os índices estavam acima do recomendado, tanto para obesas como para não obesas.

Em relação à utilização do IMC para avaliaçãa do estado nutricional e diagnóstico de sobrepeso e obesidade, a OMS reconhece que o método apresenta falha em distinguir gordura (massa gorda) de músculo (massa magra). Contudo, em geral, o IMC está correlacionado intimamente com medidas diretas da gordura corporal, sendo um forte preditor de problemas de saúde associados à obesidade, como é o caso do diabetes mellitus'22. Assim, uma vez que o IMC está associado ao aumento do risco cardiovascular em populações mais jovens, fica em aberto a questão para análise em estudos futuros, se a obesidade manteria o seu poder de predição como fator de risco cardiovascular importante também para os idosos longevos e se o IMC seria adequado para sua detecção nesta faixa etária. Isto porque um argumento que também tem sido debatido sugere que a obesidade funcionaria como um fator seletivo, levando a uma maior carga de morbimortalidade em grupos etários mais jovens, que não sobreviveriam até idades mais avançadas. Wilson e Kanne ${ }^{23}$, pesquisadores do Estudo de Framingham destacam em um artigo de revisão sobre obesidade em idosos o fato de que existiria um aumento na gordura corporal total e na adiposidade central que seria relacionado com o envelhecimento, ocorrendo até cerca dos 65 anos de idade. Caso tal aumento fosse diagnosticado, neste período, como obesidade, ele tende a vir acompanhado de diabetes mellitus ou intolerância à glicose, dentre outras morbidades e disfunções metabólicas, como foi observado em alguns dos resultados aqui descritos (hipercolesterolemia nos homens e diabetes nas mulheres). Após os 65 anos, esta condição tende a atenuar-se, principalmente se a obesidade aparece quando o indivíduo já é idoso. Ou seja, aqueles idosos longevos obesos, necessariamente, não teriam sido obesos quando jovens, mas esta condição teria evoluído em idades mais avançadas, enfraquecendo assim a carga mórbida associada à obesidade.

Estes resultados foram corroborados pelos de um estudo longitudinal dinamarquês, previamente citado $^{15}$, que encontrou que a situação marital, educação, tabagismo, obesidade, consumo de álcool e o número de doenças relatadas não estavam associados com a mortalidade de nonagenários. Apesar de não estar associada a doenças cardiovasculares e mortalidade, a obesidade neste grupo etário pode contribuir no desenvolvimento de disfunções e outras doenças, incluindo as músculo-esqueléticas, como é o caso da artrose. Portanto, não pode ser descartada a possibilidade de a obesidade ter uma forte influência na carga de morbidade, diminuição da independência funcional e na queda de qualidade de vida do idoso longevo ${ }^{15}$. Por este motivo, estudos complementares que investiguem tais relações são necessários. Ou seja, o tempo de exposiçãa aos riscos da obesidade poderia contribuir de modo significativo no impacto desta condição no desenvolvimento de doenças crônico-degenerativas.

\section{CONCLUSÕES}

A prevalência de obesidade nos idosos longevos residentes em Veranópolis-RS foi alta e similar à de países desenvolvidos. A associação entre obesidade, fatores de risco e morbidades cardiovasculares foi sexo-dependente. Os resultados obtidos tendem a ser similares a estudos previamente descritos na literatura internacional. 


\section{Agradecimentos}

À Profa. Elisabete Michelon, fundadora do Projeto Veranópolis, e toda equipe que participou da fase inicial de coleta de dados em 1996 em especial à Andréia da Costa, Ariovaldo Pires, Cristián Mercado, Luiz Pedro Marafon, Maurício Friedrich, Rodolfo Schneider e Maria Cristina Santana da Silva. À Prefeitura e Câmara de Vereadores do Município de Veranópolis, em especial aos funcionários das Secretárias da Saúde e do Trabalho e Ação Social. Aos Profs. Glauco Schneider Rolim e Ivo E. C. Jung pelo auxílio na revisão do artigo. Aos órgãos financiadores Fundação de Amparo à Pesquisa do Rio Grande do Sul (FAPERGS, processo n 98.1676. I) e pela Japan International Corporation Agency (JICA, processo $n^{\circ}$ 001/97-I) por bolsas e auxílios. Aos idosos participantes do Projeto Veranópolis, bem como aos seus familiares e cuidadores.

Conflito de interesse: não há.

\section{SUMMARY}

\section{Obesity prevalence among oldest- OLD AND ITS ASSOCIATION WITH RISK FACTORS AND CARDIOVASCULAR MORBIDITY}

OBIECTNE. to evaluate the obesity prevalence and its association with risk factors and cardiovascular morbidity in the oldest old $\geq 80$ years old) residing at the municipality of Veranópolis - RS, Brazil.

METHODOS. 196 elderly participated in the study (69 male and 127 female), 91\% of the population aged $\geq 80$ until june, 1996. For obesity evaluation and classification, we used the body mass index (BMI) and the World Health Organization (WHO) and National Health and Nutrition Examination Survey (NHANES III) criteria. The cardiovascular risk factors investigated were sex, age, systemic hypertension, hypercholesterolemia, hypertriglyceridemia, diabetes mellitus (DM) and smoking. For cardiovascular morbidities, we considered acute myocardial infarction, intermittent claudication and stroke. Waist-hip ratio $(W / H)$, regular consumption of alcoholic beverages and physical activity were investigated too. Results: the obesity prevalence was 23.3\% according to WHO (without difference between sex, $\mathrm{p}=0.124$ ) and $45.6 \%$ according to NHANES III criteria (significantly higher in female, $p=0.05$ ). Obesity associations with risk factors were sex-dependent (the obese females presented higher levels of systolic blood pressure and glucose, lower levels of HDL-c, and higher systemic hypertension and DM frequencies; while the obese males presented higher levels of diastolic blood pressure, total cholesterol, LDL-c and higher hypercholesterolemia frequency). W/H and triglycerides, as well as hypertriglyceridemia frequency, were higher in obese people.

CONCLUSIONS: the obesity prevalence was high among the long-living elderly, and its association with cardiovascular risk factors was sex-dependent. As regards morbidities, we did not observe differences between obese and non-obese people. [Rev Assoc Med Bras 2004; 50(I): 172-7]

KEY wORDS: Obesity. Cardiovascular risk factors. Cardiovascular morbidity. Long-living elderly. Oldest old.

\section{REFERÊNCIAS}

I. Chaimowicz F. A saúde dos idosos brasileiros às vésperas do século XXI: problemas, projeções e alternativas. Rev Saude Pública 1997; 3 | : | 84-200.

2. DaCruz IBM, Moriguchi EH. Projeto Veranóolis: reflexões sobre envelhecimento bem sucedido. Porto Alegre: Oficina da Longevidade; 2002. p. 138.

3. Moriguchi Y. Aspectos práticos e objetivos da medicina preventiva em Geriatria. In: Freitas EV, Py L, NeriAL, Cançado FAX, Gorzoni ML, Rocha SM. Tratado de geriatria e gerontologia. Rio de Janeiro: Guanabara Koogan; 2002. p.603-8.

4. Núcleo de Informações em Saúde. Estatísticas de saúde: mortalidade 1997. Porto Alegre: Secretaria da Saúde e do Meio Ambiente do Estado do Rio Grande do Sul; 1998. p. 264.

5. Instituto Brasileiro de Geografia e Estatística. IBGE. Censo demográfico de 1996. Disponível em: URL: http://www.ibge.gov.br.

6. Marafon LP, DaCruz IBM, Schwanke CHA, Moriguchi EH. Preditores cardiovasculares da mortalidade em idosos longevos. Cad Saúde Pública 2003; 19:799-808.

7. Michelon E, Moriguchi EH. Características da distribuição dos lipídeos plasmáticos e dos fatores de risco coronariano em indivíduos com 80 anos ou mais. Rev Med PUCRS 1996; $6: 13-23$.

8. Pena M, Bacallao J. Obesity among the poor: an emerging problem in Latin America and the Caribean. New York: Pan American Health Organization; 2000. v576, p.3-10.

9. Eberwine D. Globesity: the crisis of growing proportions. The magazine of the Pan American Health Organization 2002; 7:4.

10. Monteiro C. The epidemiologic transition in Brasil. In: Pena M, Bacallao J. Obesity among the poor: an emerging problem in latin america and caribbean. New York: Pan American Health Organization 2000; 576:3-76.
| I. Instituto Nacional de Alimentação e Nutrição. Pesquisa Nacional sobre Saúde e Nutrição (PNSN-1989) - arquivo de dados da pesquisa. Brasília: Instituto Nacional de Alimentação e Nutrição; 1990.

12. Alencar YMG, Carvalho Filho ET, Paschoal SMP, Curiati JAE, Ping WC, Litvoc J. Fatores de risco para aterosclerose em uma população idosa ambulatorial na cidade de São Paulo. Arq Bras Cardiol 2000; 74: | 8|-8.

13. Gruen DG, Connor EB. Sex differences in measures of body fat and body fat distribution in the elderly. Am J Epidemiol 1996; 1 43:898-906.

14. Abrantes MM, Lamounier JA, Colosimo EA. Overweight and obesity prevalence in Northeast and Southeast Regions of Brazil. Rev Assoc Med Bras 2003; 49: I 62-6.

15. Nybo H, Petersen HC, Gaist D, Jeune B, Andersen K, McGue M, Vaupel JW, Christensen K. Predictors of mortality in 2,249 nonagenarians-the Danish 1905-Cohort Survey. J Am Geriatr Soc 2003; 51 : 1365-73.

16. Schwanke CHA, DaCruz IBM, Leal NF, Scheibe R, Moriguchi Y, Moriguchi EH. Analysis of the association between apolipoprotein E polymorphism and cardiovascular risk factors in an elderly population with longevity. Arq Bras Cardiol 2002; 78:57। -9.

17. World Health Organization. Obesity: preventing and managing the global epidemic (report of a WHO consultation on obesity). Genebra: World Health Organization: Genebra; 1997. p.98.

18. Tavares EL, Anjos LA. Perfil antropométrico da população idosa brasileira: resultados da Pesquisa Nacional sobre Saúde e Nutrição (PNSN). Cad Saude Publica 1999; 15:759-68.

19. Centers for Disease Control and Prevention, National Center for Health Statistic. National health and nutrition examination survey (NHANES III). Atlanta: National Heart, Lung and Blood Institute; 1991.

20. Davison KK, Ford ES, Cogswell ME, Dietz $\mathrm{WH}$. Percentage of body fat and body mass index are associated with mobility limitations in people aged 70 and older from NHANES III. J Am Geriatr Soc 2002; 50: I 802-9.

21. Kuczmarski RJ, Fregal KM, Campbell SM, Johnson CL. Increasing prevalence of overweight among US adults. JAMA 1994; 272:205-II.

22. World Health Organization. The world health report 2002. Genebra: World Health Organization; 2002.

23. Wilson PW, Kannel WB. Obesity, diabetes and risk of cardiovascular disease in the elderly. Am J Geriatr Cardiol 2002; | | : | 9-25.

Artigo recebido: 16/04/03 Aceito para publicação: 17/02/04 